L. CHENG, M.-M. LI, L.-J. XIAO*, J.-H. XIE, Q.-L. ZHOU* (NANKAI UNIVERSITY, TIANJIN, P. R. OF CHINA)

Nickel(0)-Catalyzed Hydroalkylation of 1,3-Dienes with Simple Ketones

J. Am. Chem. Soc. 2018, 140, 11627-11630.

\section{Nickel-Catalyzed Enantioselective Hydroalkylation of 1,3-Dienes}

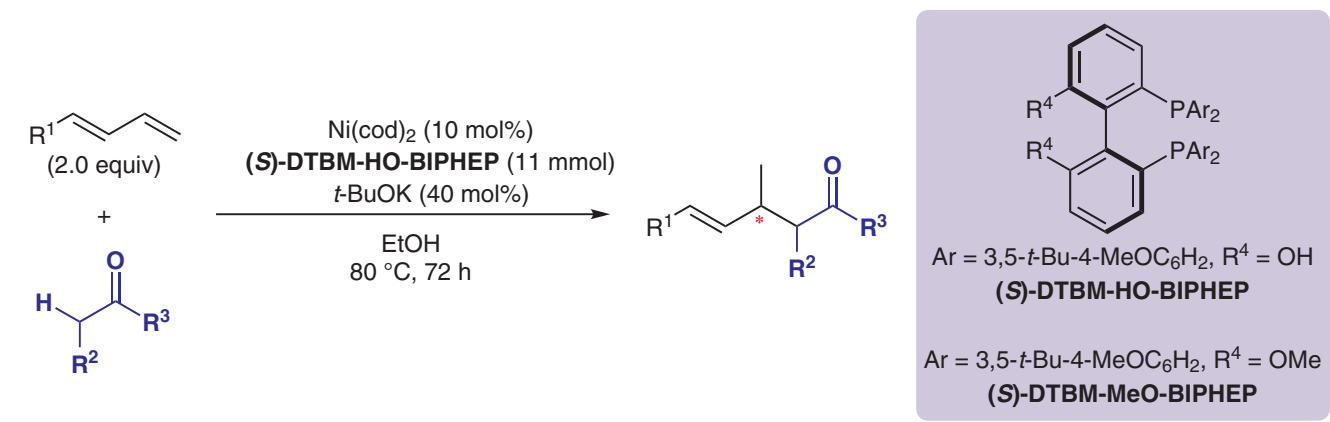

Selected examples:<smiles>CC(C=Cc1ccccc1)CC(=O)c1ccccc1</smiles>

$78 \%$ yield, er $=96: 4^{a}$<smiles>CC(C=Cc1ccccc1)C(C)C(=O)c1ccccc1</smiles>

$91 \%$ yield

$\mathrm{dr}=1.1: 1$, er $=90.5: 9.5^{\mathrm{a}}$
$78 \%$ yield, er $=96: 4^{a}$<smiles>CCOC(=O)[C@@H](c1ccccc1)C(C)/C=C/c1ccccc1</smiles>

$70 \%$ yield<smiles>CC(/C=C/c1ccccc1)CC(=O)c1ccc(-c2ccc(F)cc2F)cc1</smiles><smiles>CC(/C=C/c1ccccc1)CC(=O)c1cccs1</smiles>

$91 \%$ yield, er $=96: 4^{a}$<smiles>CC(C=CCCc1ccccc1)CC(=O)c1ccc2ccccc2c1</smiles>

$40 \%$ yield, er $=86: 14^{b}$ $d r=1.4: 1$, er $=90.5: 9.5^{a}$

a (S)-DTBM-HO-BIPHEP was employed. ${ }^{\mathrm{b}}$ Performed with (S)-DTBM-MeO-BIPHEP and reacted at $90^{\circ} \mathrm{C}$.

Derivatization:<smiles>C[C@@H](/C=C/c1ccccc1)CC(=O)c1ccc(-c2ccc(F)cc2F)cc1</smiles>

$0-20{ }^{\circ} \mathrm{C}, 24 \mathrm{~h}$
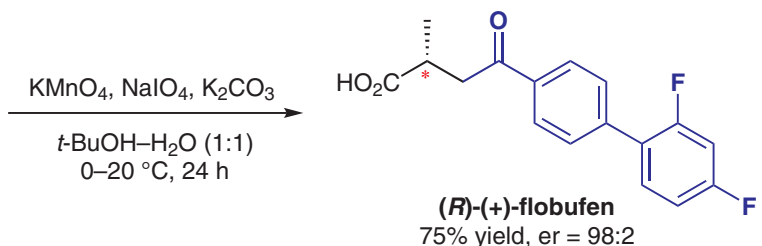

Gategory

Metal-Catalyzed

Asymmetric

Synthesis and

Stereoselective

Reactions

\section{Key words}

nickel catalysis

hydroalkylation

1,3-dienes

ketones

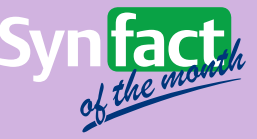

Significance: Transition-metal-catalyzed asymmetric addition of enols or enolates to unsaturated hydrocarbons remains an unanswered challenge. The authors have developed a regio- and stereoselective hydroalkylation of 1,3-dienes by nickel catalysis.
Comment: This catalytic reaction provides a wide range of $\gamma, \delta$-unsaturated ketones or esters in good yields and with high enantioselectivities. One product was easily converted into the nonsteroidal anti-inflammatory drug $(R)$-flobufen, which also exhibits immunomodulatory properties.

SYNFACTS Contributors: Hisashi Yamamoto, Wataru Muramatsu 